\title{
Introduction to the Human Genetics Symposium
}

\author{
BARTON CHILDS
}

The Johns Hopkins Hospital, Baltimore, Maryland 21205

Molecular genetics is turning up genes associated with diseases presumed hitherto to be affected only marginally, if at all, by genetic variation. Why? It is a consequence of our binary existence.That is, we all live two lives. In one, we fulfill an obligation to the species, acting as creators and transmitters of unique versions of the mosaic of human genetic variability. In this, we are each a repository of some billions of years of biologic history. In our second life, we act as effectors of that variability, pitting our individuality against an environment as richly diverse in its elements as those of the genes. In this latter role, we attempt, individually, to reconcile variation derived from two sources, each with autonomous imperatives.

The details of the organization of the DNA are being exposed by recombinant DNA techniques. Although awesomely faithful to pattern in replication, the DNA is fertile in strategies for variation and versatile in applying them in the formation of unique zygotes. But in fulfilling its adaptive missions, the DNA is entirely indifferent to the fates of its inheritors.

The mandates of human social organization are also autonomous, and although loosely designed to benefit the species, or some fragment thereof, may also be indifferent to individual outcomes.

The broad congruence of these sources of diversity is evident in Western society in a rising average duration of life. But medical science, most particularly molecular genetics (and again I emphasize in the Western world), is revealing individual incongruities in such number and ranging so broadly over diagnostic classes-including cancer, heart attack, autoimmune diseases, narcolepsy, Alzheimer's disease, affective disorder, dyslexia, and infections too (virulence must be defined in terms of the specificity of microbial molecules for those of human defense) - that we are required to transcend the limiting typology of "genetic" disease. Thus, we recognize that such a label represents merely the salience of genetic effects associated more with symptoms and burden than with genetic individuality. In its place, we may substitute a view of disease as a consequence of genetic vulnerability to selection, a vulnerability that is maximal at conception and declines throughout life. That is, since the weight of selection is commensurate with the degree of disruptive effect, the most devastating mutants will be the first to go. Further, such mutants are expressed with little regard for environmental variation which, in contrast, looms with increasing prominence and discrimination in the phenotypes of genes of lesser effect and usually of later onset. Such a gradient in selective intensity and such a reciprocal relationship to the environment is shown in Figure 1. These are data taken from reports of T-F Yu (1) on gout. The cases of earliest onset are at once the least frequent, the most severe, and the most likely to adhere to some mendelian mode of inheritance. Those in the middle are decreasingly familial with increasing age at onset, and the oldest cases, less familial still, tend to be people with hypertension being treated with diuretics that have exposed a relative incompetence, both latent and innate, to excrete uric acid. Now, you may say that, in presenting this case, I have neglected the most inherently pediatric of all attributes-development. So I hasten to add that the gradient, although continuous, is nonlinear and strongly influenced by development and aging so that the prominence of the genetic effect is greatest early in each phase of development and least at the end.

Such a genetic view of disease has implications. First, in the nexus of causes, genetic variation is likely to be represented. Second, there is an inverse relationship between the frequency of a disease and its burden as measured by shortened life, reproductive curtailment, and residual handicap, but a direct relationship between frequency and the probability of success in treatment and prevention. That is, it will always be easier to relieve pressure upon an overtaxed homeostasis than to design support for a mechanism incomplete from the start. And third, it brings into focus individuals who, through no matter how complex a concatenation of however improbable events, are, by virtue of their endowment, more-or less-likely to be numbered among those afflicted with disease. That this is not a new sentiment is illustrated by the following quote from Archibald Garrod (2): "But to our chemical individualities are due our chemical merits as well as our chemical shortcomings; and it is more nearly true to say that the factors which confer upon us our predispositions to, and immunities from the various mishaps which are spoken of as diseases, are inherent in our very chemical structure;..."

What has such a view of medicine to do with pediatrics? Why do pediatricians care about diseases with onsets well into adult life? The answer is that if we value medicine only in its most parochial applications, we don't. But if we see ourselves as

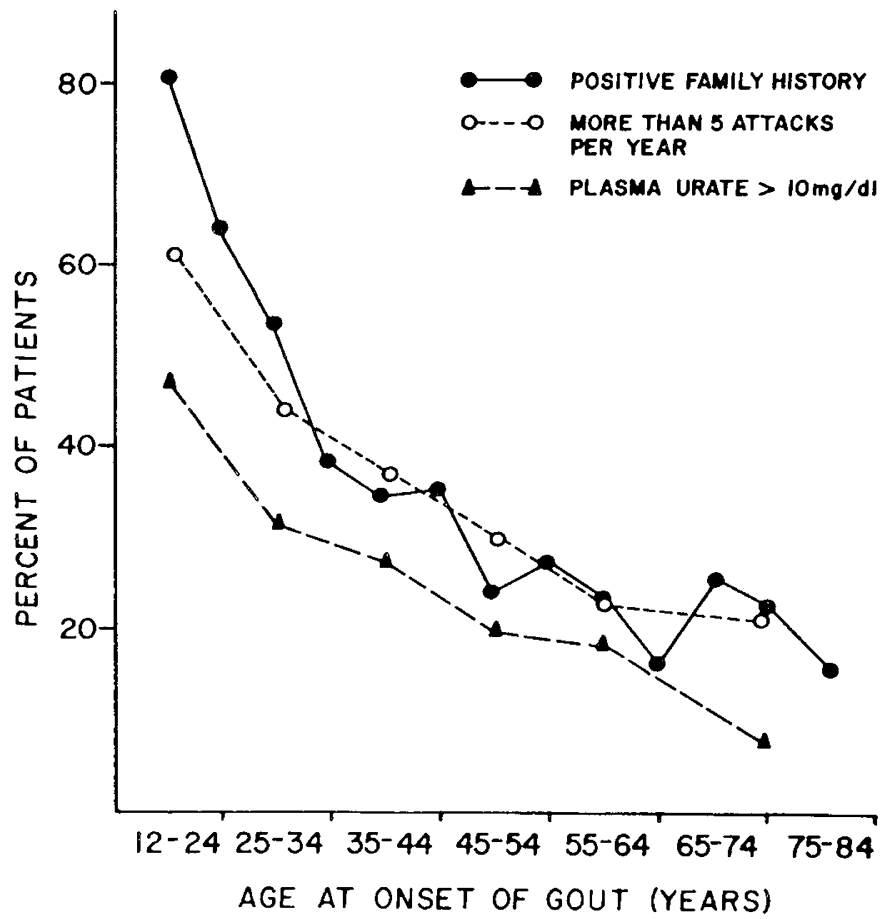

Fig. 1. Relationship of family history, severity and plasma urate levels and age at onset in gout. (T-F Yu, 1984, reprinted with permission.) 
engaged in promoting individual consonance between biologic and social organization, we do. And in fulfilling this mission we will give no less attention to individual variation than to interventions for whole populations. One hopes that the basis for choices between these alternatives will not be wholly economic, but will include some reference to the position of both diseases and individuals in the gradient of selective effect, with antenatal diagnosis, gene therapy, and insightful ingenuity in the design of treatments at one end and ordinary common sense at the other. It will be in between these polar cases that sensitivity to both individual and society will be required. In either case, this preventive need, in which genetic individuality plays so significant a part, is above all a pediatric responsibility. It is one that was perceived only dimly by our founders, but was in the forefront of the minds of those who planned this symposium. And I believe that the choice of genetics to begin today's symposium was not random. Prevention is a recurrent theme all through the day.
In what follows, Dr. Shapiro will be showing us the bountiful rewards of the methods of molecular genetics in the investigation of causes and pathogenesis of disease. Dr. Rosenberg will be telling us that however complete our knowledge of cause and pathogenesis, the need for treatment will always require unconstrained imagination, energy, and devotion. And Dr. Scriver will emphasize the pervasive role of genetic individuality in disease, both overt and potential. All of these themes are germane to the other topics on the program, so we hope they will be echoing in your minds as you listen to what others have to say.

\section{REFERENCES}

1. Yu T-F 1984 Diversity of clinical features in gouty arthritis. Semin Arthritis Rheum 13, 260-368

2. Garrod AE 1931 Inborn Factors in Disease. Oxford University Press, Oxford, p 157 